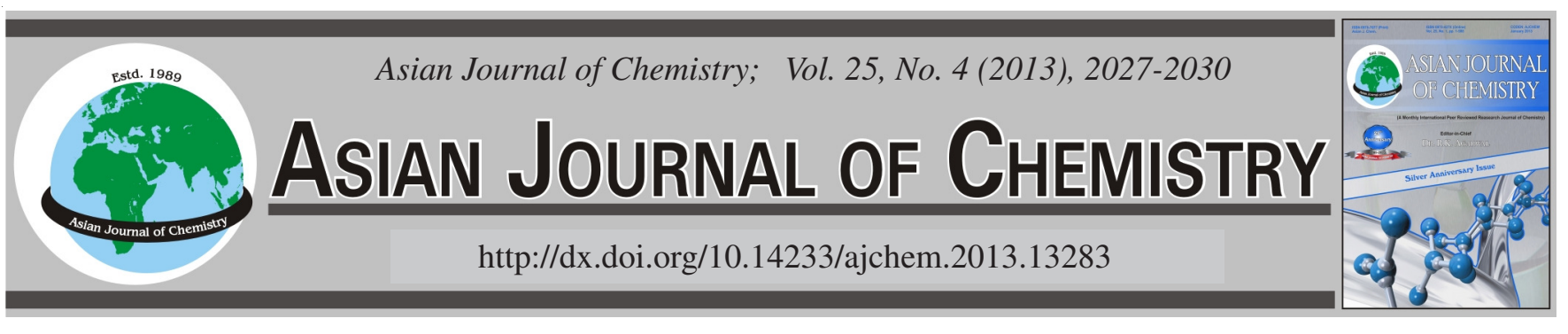

\title{
Human Intestinal Bacteria Mediate Reduction of the $N$-Oxides of Isoline and Monocrotaline to the Corresponding Parent Alkaloids
}

\author{
Jun TAng ${ }^{1,2, *}$, Zhengtao Wang $^{2,3}$, Teruaki Akao $^{4}$ and Masao Hattori ${ }^{5}$
}

${ }^{1}$ Key Laboratory of Combinatorial Biosynthesis and Drug Discovery (Wuhan University), Ministry of Education and Wuhan University School of Pharmaceutical Sciences, Wuhan 430071, P.R. China

${ }^{2}$ Department of Pharmacognosy, China Pharmaceutical University, Nanjing 210038, P.R. China

${ }^{3}$ Institute of Chinese Materia Medica, Shanghai University of Traditional Chinese Medicine, Shanghai 201203, P.R. China

${ }^{4}$ Graduate School of Medicine and Pharmaceutical Sciences, University of Toyama, 2630 Sugitani, Toyama 930-019, Japan

${ }^{5}$ Institute of Natural Medicine, University of Toyama, 2630 Sugitani, Toyama 930-019, Japan

*Corresponding author: Fax: +86 27 68759850; Tel: +86 27 68759923; E-mail: tangj0205@ gmail.com

The role of human intestinal bacteria in the biotransformation of pyrrolizidine alkaloid $N$-oxides was investigated. Two naturally-occurring hepatotoxic pyrrolizidine alkaloids, isoline and monocrotaline and their $N$-oxides were incubated with a human fecal suspension in an in vitro model, respectively. The metabolites were isolated and purified by chromatographic techniques and identified by the spectral analyses and the metabolic profiles were further analyzed by a specific TLC method. Human intestinal bacteria showed high reduction effects on the pyrrolizidine alkaloid $\mathrm{N}$-oxides but not on the resultant tertiary alkaloids, suggesting that IB may play a partial role in the cyclic conversion between each pyrrolizidine alkaloid and its $\mathrm{N}$-oxide in vivo. This evidence implied the potential risk of the pyrrolizidine alkaloid-containing Chinese medicinal herbs to human health when used in decoctions.

Key Words: Isoline $N$-oxide, Monocrotaline $N$-oxide, Hepatotoxic pyrrolizidine alkaloids, Human intestinal bacteria, Chinese medicinal herbs.

\section{INTRODUCTION}

Hepatotoxic pyrrolizidine alkaloids (PAs) are well known to be a kind of phytotoxins, with a core structure containing 1,2-unsaturated necines, e.g., retronecine or otonecine and necic acids ${ }^{1}$. Isoline and monocrotaline are two retronecinebased cyclic diester pyrrolizidine alkaloids, showing high hepatotoxicity and/or pneumotoxicity to many animal species ${ }^{2,3}$. Meanwhile, they were found to be abundant in some Chinese medicinal herbs, for example, a high level of isoline was found in Ligularia duciformis (C. Winkl.) Hand.-Mazz., which substitutes Asteris Radix as an antitussive and expectorant agent ${ }^{4}$; monocrotaline could be highly accumulated in seeds of Crotalaria assamica Benth, which have a special use in treatment of skin cancer and leukemia ${ }^{5}$. It is interesting to note that these retronecine-type pyrrolizidine alkaloids may co-exist with their corresponding $\mathrm{N}$-oxides in Chinese medicinal herbs, while more polar $N$-oxides and less tertiary alkaloids may be ingested together in decoctions by patients in Chinese medical practice ${ }^{1,6}$.

No matter what functions these $N$-oxides may have in the host plants, they may represent the detoxification products of their parent pyrrolizidine alkaloids in vivo ${ }^{6,7}$. On the other hand, some pyrrolizidine alkaloid $N$-oxides including monocrotaline $\mathrm{N}$-oxide might be reduced to their toxic parent pyrrolizidine alkaloids in human liver microsomes ${ }^{1,8}$ and the $N$-oxides of indicine and retrorsine could be reduced by the gut flora in the rabbit or rats ${ }^{9,10}$. The previous studies also showed that the gut may be the major site of the formation of PAs from their $\mathrm{N}$-oxides ${ }^{3,9}$. However, it remains unclear about what effects the human gut flora may have in the conversion between pyrrolizidine alkaloids and their $N$-oxides. Accordingly, this study reported the role of human intestinal bacteria (IB) in the metabolism of pyrrolizidine alkaloid and its $N$-oxide, using two known hepatotoxic pyrrolizidine alkaloids, that is isoline and monocrotaline, as the examples.

\section{EXPERIMENTAL}

Isoline and monocrotaline were isolated from Ligularia duciformis and Crotalaria assamica, respectively and their $\mathrm{N}$-oxides were semi-synthesized as described previously ${ }^{4,11,12}$. All chemicals and solvents were at least of AR grade. A Tabai anaerobic incubator EAN-140 (Tabai Co., Osaka, Japan) was used for anaerobic incubation. General anaerobic medium (GAM) was purchased from Nissui Co. (Tokyo, Japan). $\mathrm{K}^{+}$- 
phosphate buffer ( $\mathrm{pH}$ 7.26) was prepared as reported previously ${ }^{13}$. The modified Ehrlich reagent was prepared by dissolving 4-dimethylaminobenzaldehyde (2.0 g) with boron trifluoride ether complex $(2 \mathrm{~mL})$ in absolute ethanol $(100 \mathrm{~mL})$ freshly or kept in dark place until use. TLC was performed on a precoated silica gel $60 \mathrm{~F}_{254}$ plate $(0.25 \mathrm{~mm}$, Merck) using chloroformmethanol-28\% ammonia solution as developing system in a ratio from 8:2:0.1 to 7:3:0.1 (v/v). Column chromatography was carried out on silica gel BW-820MH or ODS (Fuji Silysia Co., Nagoya, Japan). Preparative TLC proceeded on silica gel $60 \mathrm{~F}_{254}$ plates $(0.5 \mathrm{~mm}$, Merck) and the strips were detected under a UV light or with iodine. ${ }^{1} \mathrm{H}$ and ${ }^{13} \mathrm{C}$ NMR spectra were measured with a Varian Unity Plus $500\left({ }^{1} \mathrm{H}, 500 \mathrm{MHz}\right.$; ${ }^{13} \mathrm{C}, 125 \mathrm{MHz}$ ) spectrometer (Varian, $\mathrm{CA}$ ) in $\mathrm{CDCl}_{3}$ or $\mathrm{CD}_{3} \mathrm{OD}$. EIMS was measured with a JEOL JMS-GC mate mass spectrometer (Jeol Co., Tokyo, Japan).

Preparation of a human fecal suspension: A fresh human fecal sample (1 g, collected from different part of fecal mass), obtained from a healthy subject (male, Chinese, 31 years old or Egyptian, 46 years old) with informed consent, was homogenized in GAM broth (5.9\% water solution, $20 \mathrm{~mL}$ ) or $\mathrm{K}^{+}$phosphate buffer $(50 \mathrm{mM}, 20 \mathrm{~mL})$ under anaerobic condition and the sediments were removed by filtration to give a human fecal suspension. The suspensions were used as incubation matrices in the following experiments.

Preparation of pyrrolizidine alkaloids standard stock solutions: Isoline and monocrotaline were dissolved in water with a suitable volume of $0.2 \mathrm{M}$ hydrochloric acid followed by neutralization with $0.2 \mathrm{M}$ sodium hydroxide and isoline and monocrotaline $\mathrm{N}$-oxides were dissolved in water directly to give the standard stock solutions containing $50 \mathrm{mM}$ of each compound, respectively.

Incubation of pyrrolizidine alkaloids with a human fecal suspension: All incubations were conducted according to a known in vitro model previously established in this laboratory ${ }^{13}$ with some modifications. Briefly, to the human fecal suspension $(5 \mathrm{~mL})$, standard solutions $(50 \mu \mathrm{L})$ of the PAs (including $N$-oxides) were added in aliquots, respectively and then incubated at $37{ }^{\circ} \mathrm{C}$ in the anaerobic incubator for more than $6 \mathrm{~h}$. Meanwhile, various controls including a control without PA, a control in GAM broth without feces or that under aerobic condition were examined in parallel. A $100 \mu \mathrm{L}$ of portion was taken out at intervals of at least $1 \mathrm{~h}$ and extracted with $100 \mu \mathrm{L}$ of $n$-butanol (saturated with water and adjusted to $\mathrm{pH} 8-9$ with $28 \%$ ammonia solution). After centrifugation at $10,000 \mathrm{~g}$ for $3 \mathrm{~min}, 5 \mu \mathrm{L}$ of the $n$-butanol layer, the corresponding aqueous layer as well as different standard solution was applied to TLC, respectively. Afterwards, three steps proceeded for the visualization and detection of the substrates and possible metabolites, that is, first oxidized with iodine and/or $30 \%$ hydrogen peroxide (only for parent alkaloids), then dehydrogenated with acetic anhydride and finally reacted with Ehrlich reagent. All steps need heating except iodine exposure. The appearance of a distinct magenta colour (Ehrlich colour) was regarded as the positive reaction. In this condition, the limits of detection could be $0.1 \mu \mathrm{g}$ for isoline and $0.3 \mu \mathrm{g}$ for monocrotaline. All experiments were conducted in triplicate.
For the identification of metabolites, a scaled-up incubation was performed with a human fecal suspension $(50 \mathrm{~mL})$ containing each $\mathrm{N}$-oxide $(0.5 \mathrm{mM})$ at $37^{\circ} \mathrm{C}$ for $5 \mathrm{~h}$. The final mixtures were then extracted with $n$-butanol and separated through repeated column chromatography and/or preparative TLC.

\section{RESULTS AND DISCUSSION}

Characterization of the metabolites of two pyrrolizidine alkaloid $\mathbf{N}$-oxides: After the scaled-up incubations with two PA $N$-oxides, metabolite $\mathbf{1}(5 \mathrm{mg})$ and $2(3 \mathrm{mg})$ were obtained with a rate of 51 and $38 \%$, respectively. As shown below, metabolite $\mathbf{1}$ was obtained from isoline $N$-oxide as a white needle. The EIMS showed a molecular ion peak $[\mathrm{M}]^{+}$at $\mathrm{m} / \mathrm{z}, 395$ as a base. Metabolite 2 was obtained from monocrotaline $\mathrm{N}$-oxide as a fan-shaped crystal. The EIMS showed a molecular ion peak $[\mathrm{M}]^{+}$at $m / z$ 325. The MS fragmentation pattern for each compound was similar to that of isoline and monocrotaline, respectively. Meanwhile, both metabolites gave the same characteristic ions of a retronecinetype necine base at $\mathrm{m} / \mathrm{z} 136,120,93$ and 80 . The ${ }^{1} \mathrm{H}$ and ${ }^{13} \mathrm{C}$ NMR spectral data of metabolites $\mathbf{1}$ and $\mathbf{2}$, were in agreement with those reported for isoline $\mathrm{e}^{14}$ and for monocrotaline ${ }^{15}$, respectively. The co-TLC analyses with authentic samples also confirmed $\mathbf{1}$ and $\mathbf{2}$ as isoline and monocrotaline, respectively (Fig. 1). The lower yield rate of $\mathbf{2}$ may be ascribed to its higher hydrophilicity and poor partition in $n$-butanol.

Metabolite 1 A white needle (hexane/acetone), m.p. 168$169{ }^{\circ} \mathrm{C}$. EIMS $m / z(\%): 395\left(\mathrm{M}^{+}, 100\right), 366\left(\mathrm{M}^{+}-\mathrm{CH}_{3} \mathrm{CH}_{2}, 8\right)$, $335\left(\mathrm{M}^{+}-\mathrm{CH}_{3} \mathrm{COOH}, 15\right), 320\left(\mathrm{M}^{+}-\mathrm{CH}_{3} \mathrm{COOH}-\mathrm{CH}_{3}, 10\right), 309$, $308\left(\mathrm{M}^{+}-\mathrm{CH}_{3} \mathrm{CO}-\mathrm{CO}_{2}, 98\right), 291\left(309-\mathrm{H}_{2} \mathrm{O}, 11\right), 236$ (308$\left.\mathrm{CH}_{3} \mathrm{COCH}_{2} \mathrm{CH}_{3}, 16\right), 220$ (308- $\left.\mathrm{CH}_{3} \mathrm{COCHCH}_{3} \mathrm{OH}, 50\right), 138$, 136, 120, 118, 108, 95, 93, 83, 80. ${ }^{1} \mathrm{H} \mathrm{NMR}\left(\mathrm{CDCl}_{3}, 500 \mathrm{MHz}\right)$

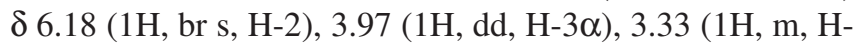

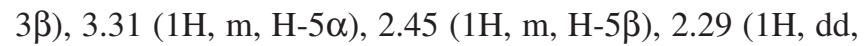

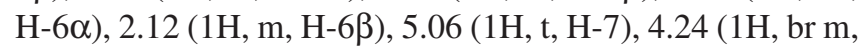

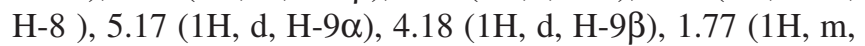

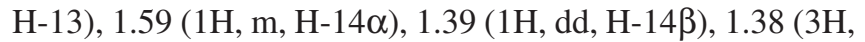
s, H-18), 1.21 (3H, d, H-19), 1.59 (2H, m, H-20), 0.80 (3H, t, $\mathrm{H}-21), 2.03$ (3H, s, $\left.\mathrm{CH}_{3} \mathrm{CO}\right) .{ }^{13} \mathrm{C} \mathrm{NMR}\left(\mathrm{CDCl}_{3}, 125 \mathrm{MHz}\right) \delta$ : 131.2 (C-1), 135.9 (C-2), 63.4 (C-3), 53.2 (C-5), 34.5 (C-6), 76.8 (C-7), 77.4 (C-8), 60.6 (C-9), 172.2 (C-11), 83.5 (C-12), 37.2 (C-13), 39.4 (C-14), 78.8 (C-15), 176.1 (C-16), 14.8 (C-18), 15.8 (C-19), 33.3 (C-20), 7.4 (C-21), $170.0\left(\right.$ (OCH$\left._{3}\right)$, $21.1\left(\mathrm{CO}^{\mathrm{C}} \mathrm{H}_{3}\right)$.

Metabolite 2 A white fan-shaped crystal (chloroform), m.p. $202{ }^{\circ} \mathrm{C}$. EIMS $\mathrm{m} / \mathrm{z}(\%): 325\left(\mathrm{M}^{+}, 23\right), 281\left(\mathrm{M}^{+}-\mathrm{CO}_{2}, 7\right)$, 237 (281- $\left.\mathrm{CH}_{3} \mathrm{COH}, 75\right), 236$ (100), 193 (237- $\left.\mathrm{CH}_{3} \mathrm{COH}, 14\right)$, 165 (193- $\left.\mathrm{CH}_{3} \mathrm{CH}, 6\right), 137,136,120,119,94,93,80 .{ }^{1} \mathrm{H}$ NMR $\left(\mathrm{CDCl}_{3}, 500 \mathrm{MHz}\right) \delta: 6.04$ (1H, br.s, H-2), $3.90(1 \mathrm{H}, \mathrm{d}, \mathrm{H}-$

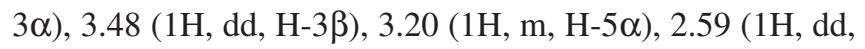
H-5ß), 2.08 (2H, m, H-6), 5.05 (1H, m, H-7), 4.38 (1H, br m,

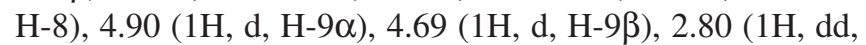
14-H), 1.44 (3H, s, H-17), 1.35 (3H, s, H-18), 1.22 (3H, d, H19). ${ }^{13} \mathrm{C} \mathrm{NMR}\left(\mathrm{CDCl}_{3}, 125 \mathrm{MHz}\right) \delta: 132.7$ (C-1), $134.3(\mathrm{C}-2)$, 61.3 (C-3), 53.6 (C-5), 33.5 (C-6), 75.1 (C-7), 76.8 (C-8), 60.5 (C-9), 174.0 (C-11), 76.7 (C-12), 78.7 (C-13), 44.3 (C14), 173.5 (C-15), 21.9 (C-17), 17.6 (C-18), 13.6 (C-19). 


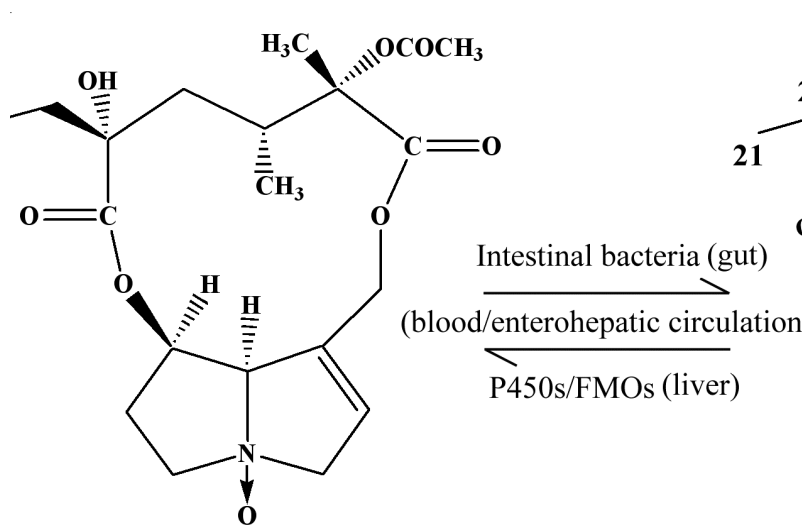

Isoline $\mathrm{N}$-oxide, $\mathrm{C}_{20} \mathrm{H}_{29} \mathrm{NO}_{8}$

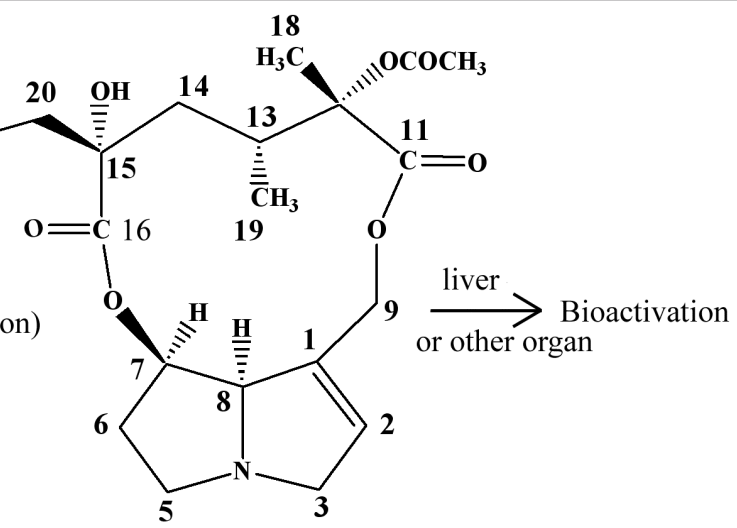

Isoline, $\mathrm{C}_{20} \mathrm{H}_{29} \mathrm{NO}_{7}$

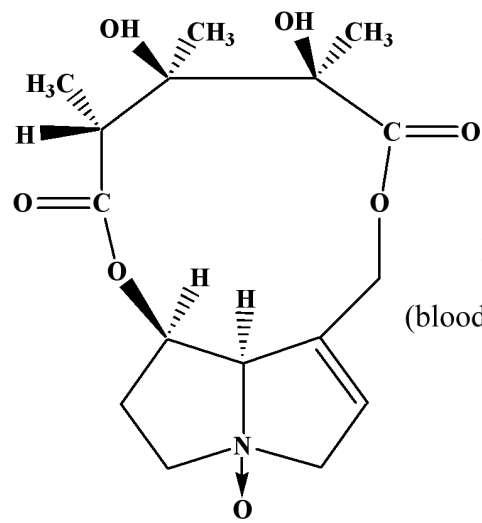

Monocrotaline N-oxide, $\mathrm{C}_{16} \mathrm{H}_{23} \mathrm{NO}_{7}$

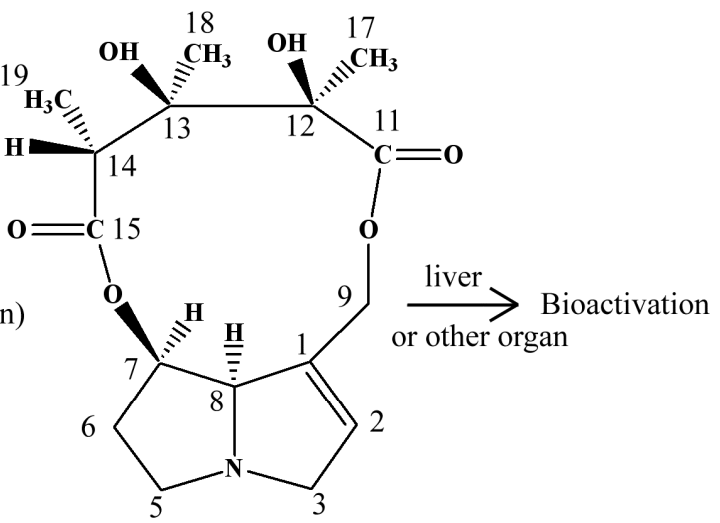

Monocrotaline, $\mathrm{C}_{16} \mathrm{H}_{23} \mathrm{NO}_{6}$

Fig. 1. Reduction of isoline or monocrotaline $N$-oxide to the corresponding tertiary alkaloid by intestinal bacteria and other metabolic pathways mediated by liver enzymes

Metabolic profiles of two pyrrolizidine alkaloid $N$ oxides and their parent alkaloids by human intestinal bacteria: The biotransformation of two hepatotoxic PAs and their $\mathrm{N}$-oxides were conducted in a previously described model for in vitro metabolism involved by intestine bacteria, which could better simulate an in vivo condition ${ }^{13}$. Moreover, a specific TLC method employing Ehrlich reagent was developed for the examination of PAs and their metabolites, whose specificity was based on the unique pyrrolic derivatives from PAs or their metabolites and the associated nucleophilic reaction with the Ehrlich reagent. As a result, isoline $N$-oxide and monocrotaline $\mathrm{N}$-oxide were completely transformed within 4.5 and $3.0 \mathrm{~h}$ into their tertiary bases, respectively (Fig. 2A, C). The two metabolites had higher $\mathrm{R}_{\mathrm{f}}$ values $(0.77$ and 0.62$)$ than their corresponding $N$-oxide substrates ( 0.47 and 0.18 ), showing a remarkable loss in the polarity after conversion. TLC results from the various controls indicated that the change was mainly mediated by IB. Among them, the control without PA showed no other spots with the same $\mathrm{R}_{\mathrm{f}}$ values and colours as either $\mathrm{N}$-oxide substrates or their metabolites (Fig. 2E). The control without IB showed a slight decrease of two $N$-oxide substrates and the corresponding formation of parent PAs, however, this process was so slow that large amount of $\mathrm{N}$-oxides remained unchanged even after more than $10 \mathrm{~h}$, in which both $N$-oxides were almost stable within the first $3 \mathrm{~h}$ incubation, whereas at least $4 \mathrm{~h}$ under aerobic condition (Fig. 2B, D).
Conversely, both isoline and monocrotaline were found unchanged during $120 \mathrm{~h}$ incubation with human intestine bacteria. By TLC, no other spots except PA substrates were detected with positive Ehrlich colour (Fig. 2E). Moreover, this study has also examined the different human race and rat strain and/or used clivorine (an otonecine-type PA) as substrate and showed same results too (data not shown here). In contrast to the liver in which monocrotaline, isoline and clivorine can be bioactivated or detoxified ${ }^{3,7,16}$, the gut flora may not be a metabolic site for these tertiary alkaloids (Fig. 1).

Taken together, the resultant metabolites of two PA $N$ oxides by human IB were the less polar but more stable tertiary alkaloids, which may more likely be re-absorbed from the intestinal tract or through enterohepatic circulation (Scheme-I). The metabolic rates showed somewhat different for two $N$-oxides by TLC, suggesting that this conversion may be substrate-dependent. Even though two substrates showed a slight chemical decomposition under anaerobic condition, they did not change so extensively and rapidly as in the presence of IB prior to excretion. These evidences indicated that human IB play an essential role in the rapid and irreversible reduction of PA $N$-oxides in the intestinal tract, which supported that the detoxification of PAs via $\mathrm{N}$-oxidation may be reversible and oral ingestion of PAs and their $\mathrm{N}$-oxides through a decoction of Chinese herbs may pose a potential risk on human health ${ }^{1,6,17}$. 


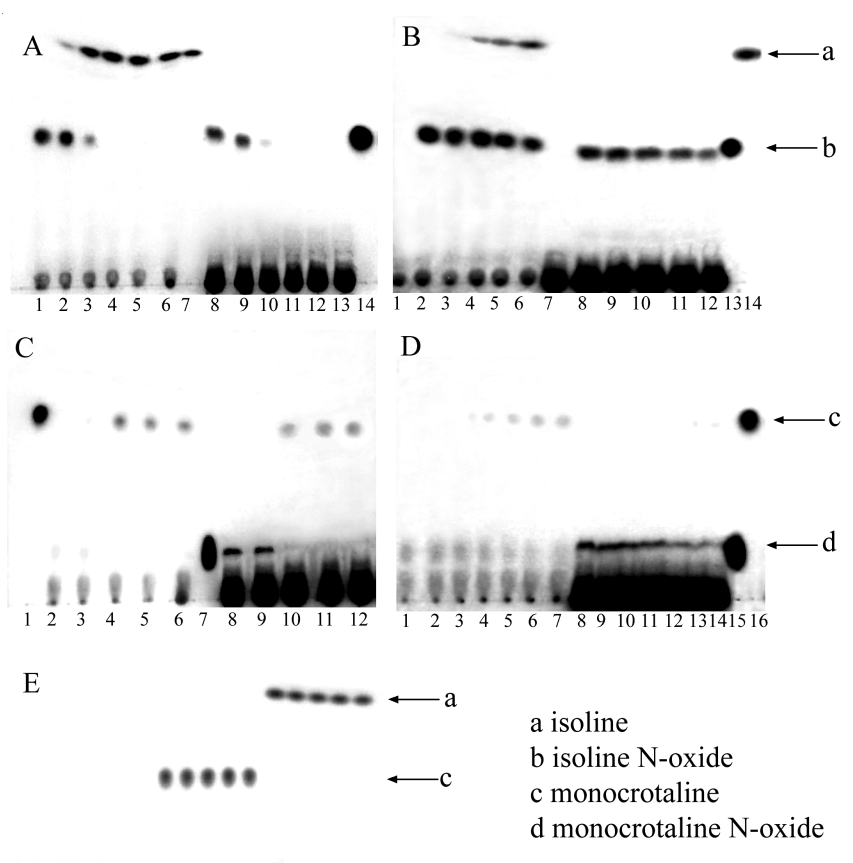

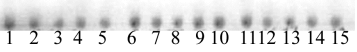

Fig. 2. Representative TLC profiles of metabolism of isoline, monocrotaline and their $N$-oxides by human intestinal bacteria. (A) Incubation of isoline $N$-oxide with human fecal suspension. 1-6 $n$-butanol extracts after incubation for $0,1,3,4.5,6,8.5 \mathrm{~h} ; 7$ isoline; 8 -13 the corresponding $\mathrm{H}_{2} \mathrm{O}$ layers after $n$-butanol extraction; 14 isoline $N$ oxide; (B) Control incubation of isoline $N$-oxide in GAM broth without feces. 1,7 $n$-butanol extract and the corresponding $\mathrm{H}_{2} \mathrm{O}$ layer of control without PA; 2-6 $n$-butanol extracts after incubation for $0,3,7,11,19 \mathrm{~h} ; 8-12$ the corresponding $\mathrm{H}_{2} \mathrm{O}$ layers after $n$-butanol extraction; 13 isoline $N$-oxide; 14 isoline; (C) Incubation of monocrotaline $N$-oxide with human fecal suspension. 1 monocrotaline; 2-6 $n$-butanol extracts after incubation for $0,1,3$, $4.5,6$ h; 7 monocrotaline $N$-oxide; 8-12 the corresponding $\mathrm{H}_{2} \mathrm{O}$ layers after $n$-butanol extraction; (D) Control incubation of monocrotaline $N$-oxide in GAM broth without feces. 1-7 $n$-butanol extracts after incubation for $0,3,7,11,19,24,31 \mathrm{~h} ; 8-14$ the corresponding $\mathrm{H}_{2} \mathrm{O}$ layers after $n$-butanol extraction; 15 monocrotaline $N$-oxide; 16 monocrotaline; (E) Incubation of isoline or monocrotaline with human fecal suspension. 1-5 control incubates (without PAs) for $0,25,50,75,120 \mathrm{~h}$ in sequence; 6-10 monocrotaline incubates for $0,25,50,75,120 \mathrm{~h}$ in sequence; 11 15 isoline incubates for $0,25,50,75,120 \mathrm{~h}$ in sequence

\section{Conclusion}

In this study, the biotransformation of two hepatotoxic PAs and their $N$-oxides were conducted using a convenient and non-invasive in vitro model. Moreover, a specific TLC method employing Ehrlich reagent was developed for the examination of PAs and their metabolites. The results revealed that two PA $N$-oxides but not their parent alkaloids could be rapidly and extensively transformed by human IB, which may be partially responsible for the cyclic conversion between each PA and its $N$-oxide and play an essential role in the toxic outcomes of the PAs or PA-containing Chinese medicinal herbs in Chinese medical practice.

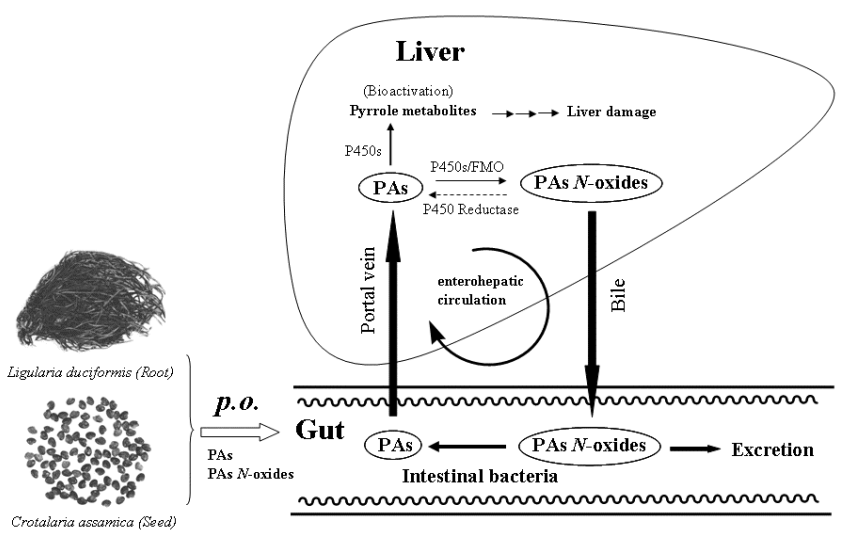

Scheme-I: Proposed cyclic conversion between PA and its $N$-oxide through enterohepatic circulation in vivo and a partial role of the intestinal bacteria. The $N$-oxides of isoline and monocrotaline derived from two hepatotoxic Chinese herbs could be completely and rapidly reduced to their parent alkaloids by human intestinal bacteria, which may be reabsorbed into the liver together with the originally contained tertiary PAs and suffered further bioactivation. Some $N$-oxides formed in liver may be excreted into the gut again with biles

\section{ACKNOWLEDGEMENTS}

This research grants from the Natural Science Foundation of China (Nos. 31270401 and 39825129) and Wuhan University Start-up Funding for the Introduced Talents to Dr. Jun Tang (306276214) are greatly acknowledged.

\section{REFERENCES}

1. J. Tang and M. Hattori, Acta Pharm. Sin., 46, 762 (2011).

2. R.J. Huxtable, Pharmacol. Ther., 47, 371 (1990).

3. J. Tang, T. Akao, N. Nakamura, Z.T. Wang, K. Takagawa, M. Sasahara and M. Hattori, Drug Metab. Dispos., 35, 1832 (2007).

4. J. Tang, M. Zhang, Z.T. Wang, T. Akao, N. Nakamura and M. Hattori, J. Anal. Toxicol., 28, 11 (2004).

5. L. Jiang, M. Cheng, Q. Gao and J. Tang, China Pharm., 13, 1743 (2010).

6. E. Roeder, Pharmazie, 55, 711 (2000).

7. P.P. Fu, Q.S. Xia, G. Lin and M.W. Chou, Drug Metab. Rev., 36, 1 (2004).

8. Y.P. Wang, J. Yan, P.P. Fu and M.W. Chou, Toxicol. Lett., 155, 411 (2005).

9. G. Powis, M.M. Ames and J.S. Kovach, Cancer Res., 39, 3564 (1979).

10. P.S. Chu, M.W. Lame and H.J. Segall, Arch. Toxicol., 67, 39 (1993).

11. J. Tang, M. Zhang, Z.T. Wang, T. Akao, N. Nakamura, M. Hattori and G.J. Xu, Chin. J. Nat. Med., 1, 21 (2003).

12. J. Tang, M. Zhang, Z.T. Wang, T. Akao, N. Nakamura and M. Hattori, J. Chin. Pharm. Univ., 34, 499 (2003).

13. L.Q. Wang, M.R. Meselhy, Y. Li, G.W. Qin and M. Hattori, Chem. Pharm. Bull., 48, 1606 (2000).

14. L. Susag, M. Parvez, S. Mathenge and M.H. Benn, Phytochemistry, 54, 933 (2000).

15. C.G. Logie, M.R. Grue and J.R. Liddell, Phytochemistry, 37, 43 (1994).

16. S.R. Dueker, M.W. Lame, D. Morin, D.W. Wilson and H.J. Segall, Drug Metab. Dispos., 20, 275 (1992).

17. M.W. Chou, Y.P. Wang, J. Yan, Y.C. Yang, R.D. Beger, L.D. Williams, D.R. Doerge and P.P. Fu, Toxicol. Lett., 145, 239 (2003). 